\title{
VARIABILIDAD TEMPORAL EN LA PRODUCCIÓN DE ARTEFACTOS DE ADORNO PERSONAL EN PATAGONIA CONTINENTAL: ANÁLISIS A PARTIR DEL SITIO POBLACIÓN ANTICURA (PROVINCIA DE RÍO NEGRO, ARGENTINA)
}

SABRINA LEONARDTa

\section{RESUMEN}

Este trabajo se presenta como un primer paso para evaluar el papel de los artefactos de adorno personal en el contexto de creciente interacción social que caracteriza a los últimos 2.000 años en Patagonia, partiendo del interrogante de si estos objetos reflejan una instancia más de la presencia de códigos comunicacionales compartidos a escala regional. Se propone como punto de partida evaluar las características del registro de artefactos de adorno personal a través de los distintos momentos del Holoceno en Patagonia continental. Se parte del análisis de las características y distribución temporal de las cuentas y pendientes recuperados en el sitio arqueológico Población Anticura (provincia de Río Negro, Argentina) y se comparan las tendencias observadas a escala local con la información disponible en la bibliografía arqueológica de Patagonia continental. Los resultados obtenidos dan cuenta de la elaboración de adornos personales desde momentos tempranos (Holoceno temprano y medio), evidenciando una mayor variabilidad en relación al registro de contextos datados en el Holoceno tardío. Para estos momentos se observa, principalmente a partir de los últimos 2.000 años, el predominio de cuentas respecto de pendientes y una mayor homogeneidad manifestada en el predominio de morfologías de tipo circular/subcircular y en la selección de la valva como materia prima dominante para su elaboración.

PALABRAS CLAVE: adornos personales, Patagonia continental, Holoceno, cuentas.

\section{TEMPORAL VARIABILITY IN THE PRODUCTION OF BODY ORNAMENTS IN CONTINENTAL PATAGONIA: ANALYSIS FROM POBLACION ANTICURA SITE (RIO NEGRO PROVINCE, ARGENTINA)}

\begin{abstract}
This paper is a first step to evaluate the role played by body ornaments in the context of the increasing social interaction that characterized the last 2.000 years in Patagonia. The initial question was if these objects, between others, were another instance of the presence of communication codes regionally shared. In so doing, the characteristics of body ornaments record were evaluated for the different moments of the Holocene in continental Patagonia. The starting point was the analysis of


the characteristics and temporal distribution of beads and pendants recovered in archaeological site Población Anticura (Río Negro province, Argentina). Those results were then compared with regional trends obtained from the information available in archaeological bibliography of continental Patagonia. The results show that body ornaments are present in archaeological record since Early and Middle Holocene, showing greater variability in relation to sets dated in Late Holocene contexts. For these last moments, mainly since the last 2.000 years, the predominance of beads and homogeneity in the manufacture are manifested in the prevalence of circular/sub-circular morphologies and in the selection of shell as the dominant raw material for ornaments production.

KEY WORDS: body ornaments, continental Patagonia, Holocene, shell beads

\section{INTRODUCCIÓN}

Ciertos artefactos, a partir de sus características estilisticas, pueden transportar mensajes de distinto tipo entre miembros o grupos distantes que no están en situaciones de contacto verbal constante (Wobst, 1977). Varios autores han planteado escenarios de este tipo para explicar los patrones estilísticos y distribucionales de diferentes objetos artísticos (Barton et al. 1994; Gamble, 1982; Mc Donald, 2008; Stiner, 2014, entre otros). Dentro de esta categoría se incluyen los adornos corporales que, en tanto artefactos generalmente elaborados con el fin expreso de ser vistos, tienen como característica dominante la expresión a través de una imagen visual (White, 1993; Taborin, 2004; Fiore, 2011). Estos artefactos pueden jugar un papel importante en la comunicación de distinto tipo de información social a distintas escalas: desde expresar estados particulares del portador tales como estatus, edad o prestigio, como portar mensajes de integración o diferenciación social estableciendo límites o creando lazos interpersonales (Wiessner, 1983; White, 1993; Taborin, 2004; Whallon, 2006; Kuhn \& Stiner, 2007). Las cuentas suelen ser un artefacto de adorno corporal frecuentemente recuperado en el registro arqueológico de Patagonia (véase Zubimendi, 2015). Su presencia en momentos tardíos está asociada tanto a contextos mortuorios, formando parte de ajuares (por ejemplo Cassiodoro, 2005; Della Negra \& Ibañez Saint Paul, 2012), como a contextos habitacionales (por ejemplo Prates, 2008; Fernández \& Ramos, 2009). Sin embargo, aún no es claro el papel que tuvieron estos artefactos en el pasado.

Los últimos 2.000 años en Patagonia se caracterizan por un incremento en la densidad poblacional y la ocupación efectiva de la mayor parte de los espacios y ambientes disponibles (Borrero, 1994-1995, 2001), en algunos casos correlacionado con una reducción de los rangos de movilidad residencial (Borrero, 1994-1995; Goñi, 2000; Gómez Otero, 2006). Paralelamente, la amplia distribución espacial que alcanzan distintos ítems tales como obsidianas, moluscos, placas grabadas y el arte rupestre da cuenta de la existencia de amplias redes de circulación de materiales e interacción entre las poblaciones que habitaron la región (Borrero, 1994-1995, 2001; Gómez Otero, 2006; Bellelli et al. 2008; entre otros). En este sentido, la gran dispersión espacial que alcanza para momentos tardíos la Tendencia Abstracto Geométrico Compleja (TAGC) (Gradin, 1999), en el arte rupestre y mobiliar, ha sido interpretada por varios investigadores como la manifestación de un código visual compartido a escala regional que habría funcionando como un sistema de transmisión de información entre poblaciones (véase Belardi, 2004; Fiore \& Borella, 2010; Scheinsohn, 2011 entre otros).

Considerando la potencialidad de los adornos corporales como medios de comunicación visual y teniendo en cuenta el contexto de interacción entre las poblaciones que habitaron la región durante los últimos 2.000 años, este trabajo se enmarca en un proyecto de investigación que apunta a evaluar el papel que jugaron las cuentas a escala regional en Patagonia para momentos tardíos. En este sentido, se parte de la pregunta de si es posible que, de manera similar a lo postulado para la TAGC, las cuentas reflejen una instancia más de la existencia de códigos comunicacionales compartidos a escala regional en Patagonia continental. En principio, para evaluar esta 
posibilidad es necesario reconocer: a) la existencia de diferencias en la frecuencia y características de los artefactos de adorno corporal en Holoceno tardío respecto de momentos anteriores; b) la convergencia, durante momentos tardíos, de formas, tamaños y/o materias primas que den cuenta de la existencia de patrones estéticos compartidos a través de amplias áreas, ya que la comunicación de un mensaje requiere cierto grado de estandarización que permita su codificación y decodificación (Fiore, 2011; Stiner, 2014) y c) la producción local de estos artefactos en distintos sectores de Patagonia, en función de que el código necesita ser replicable (Kuhn \& Stiner, 2007). Este último aspecto comenzó a evaluarse en un trabajo anterior a partir del análisis de contextos de producción de cuentas en sitios arqueológicos del bosque del noroeste de Patagonia (Leonardt, 2014).

El presente trabajo tiene por objetivo realizar un primer abordaje de estos tres aspectos en conjunto. Para ello, se analizan los adornos corporales y desechos de manufactura de cuentas de valva del sitio Población Anticura (suroeste de Río Negro, Argentina) cuya secuencia ocupacional se remonta al Holoceno temprano (Bellelli et al. 2013), a fin de comparar las tendencias observadas a escala local con los patrones registrados a escala regional en Patagonia continental.

\section{EL ESTUDIO DE CUENTAS Y PENDIENTES EN PATAGONIA}

La información sobre cuentas y pendientes en la bibliografía arqueológica de Patagonia es muy dispar. En la mayoría de los casos su presencia sólo se menciona como parte del inventario de materiales recuperados en los sitios, muchas de las veces con descripciones someras y sin fotografías o dibujos asociados (por ejemplo Gradin et al. 1976; Onetto, 1986-1987; Boschín, 2009; Franco et al. 2012). No obstante, en los últimos años se han desarrollado una serie de trabajos que abordan el análisis de este registro desde distintas perspectivas. En algunos casos se ha apuntando a su caracterización tecnológica, planteando posibles cadenas operativas y discutiendo las posibles fuentes de aprovisionamiento de materias primas y sus implicancias (Cimino, 2007; Prates, 2008; Crivelli Montero \& Ramos, 2009; Fernández \& Ramos, 2009; Zubimendi \& Moreno, 2014). Otro conjunto de trabajos abordan su relación con el registro funerario (Cassiodoro, 2005; Cassiodoro \& García Guraieb, 2009; Della Negra \& Ibañez Saint Paul, 2012).

En una escala espacial amplia, algunos estudios evalúan la existencia de patrones regionales, tal como el trabajo de Fernández para los "hallazgos especiales" de la cuenca inferior del río Limay (Fernández, 2009) en donde se postula un incremento del uso de artefactos de adorno a partir de los últimos 2000 años AP en relación con una demografía en crecimiento (Fernández, 2009). A su turno, los análisis de la distribución de artefactos malacológicos, entre ellos cuentas, realizados en Patagonia central han permitido postular un patrón de distribución asociado a su obtención indirecta, a partir de circuitos de interacción a escala regional (Zubimendi \& Ambrústolo, 2011). En este contexto, se ha planteado que los moluscos marinos y las cuentas, usadas como adorno corporal, pudieron haber tenido una fuerte carga simbólica (Zubimendi, 2010; Zubimendi \& Ambrústolo, 2011). Asimismo, para la zona del canal de Beagle (Tierra del Fuego) caben destacar los análisis de Fiore (2012) respecto de los cambios en los patrones y ritmos de producción y decoración del arte mobiliar óseo, dentro del que se incluyen las cuentas, durante el Holoceno.

Siguiendo esta línea que considera una escala espacial amplia, en un trabajo anterior se abordó el análisis de la identificación de contextos de producción local de cuentas de valva en el bosque del noroeste de Patagonia (Leonardt, 2014). El mismo partió del desarrollo de un programa de arqueología experimental que permitió definir un patrón de desechos de manufactura de cuentas, esperables en situaciones de producción no especializada. Así, fue posible identificar contextos de elaboración local de adornos de valva en dos sitios del bosque del noroeste de Patagonia, uno de ellos, el que aquí se trata: Población Anticura (Leonardt, 2014).

\section{LAS INVESTIGACIONES EN EL VALLE DEL RÍO MANSO INFERIOR: EL SITIO POBLACIÓN ANTICURA}

El valle del río Manso inferior se localiza en el extremo suroeste de la provincia de Río Negro, 
en ambiente de bosque andino-patagónico. La cronología y características de los sitios en el área se integran dentro del marco regional de ocupación de los ambientes boscosos de Norpatagonia para el cual se sostiene una mayor intensidad de uso por parte de los cazadores-recolectores entre $1.200 \mathrm{y}$ 200 años AP (Fernández et al. 2013). La mayoría de los sitios allí registrados corresponden a aleros con pinturas rupestres atribuibles a la TAGC y a una variante regional denominada Modalidad del Ámbito Lacustre Boscoso, propia de los ambientes boscosos de Patagonia septentrional (Podestá et al. 2009). Esto, sumado a los patrones decorativos de placas grabadas y cerámica registrados en el área, permite sostener la existencia de un mismo código visual que vincula esta área con las poblaciones terrestres a ambos lados de la cordillera de los Andes y con la estepa (Bellelli et al. 2008; Podestá et al. 2009).

Población Anticura se localiza en el valle del río Manso inferior (suroeste de Río Negro) y es un alero rocoso que posee evidencias de ocupación en distintos momentos del Holoceno, constituyendo el registro con las evidencias más antiguas para el valle y el área vecina de la Comarca Andina del Paralelo $42^{\circ}$ (Bellelli et al. 2013). Las excavaciones realizadas en este sitio permitieron definir una secuencia de ocupaciones asignables a distintos segmentos temporales en relación con la cronología general establecida para el valle (Fernández et al. 2013). Los niveles más tempranos del sitio están datados en alrededor de 8.000 años AP y se caracterizan por la presencia de escasos restos materiales asociados a fogones (Bellelli et al. 2013). Otro conjunto de ocupaciones ha sido recientemente fechado en alrededor de 4.700 años AP (P. Fernández, com. pers. 2014) y determinaría el uso del sitio en momentos correspondientes al Holoceno medio, también con escasos restos asociados. Para el Holoceno tardío se pueden establecer dos momentos. Entre los 3.350 y 2.270 años AP (Holoceno tardío inicial) se registra un grupo de ocupaciones relacionadas principalmente con eventos mortuorios y caracterizadas por una baja frecuencia de evidencia arqueológica y la presencia de restos óseos humanos correspondientes a dos individuos: un subadulto datado en $3.180 \pm 30$ años AP y un adulto datado en $2.960 \pm 25$ años AP respectivamente (Fernández et al. 2013).
Finalmente, el uso más tardío del sitio comprende un lapso que va entre 1.200 y 200 años AP (Holoceno tardío final) y concentra las evidencias de manufactura local de cuentas de valva (Leonardt, 2014) y la mayor parte de la evidencia arqueológica recuperada (Fernández et al. 2010).

\section{METODOLOGÍA}

En este trabajo se integra la totalidad de los artefactos de adorno corporal $(\mathrm{N}=27)$ y de los fragmentos de valva $(\mathrm{N}=630)$ recuperados en el sitio Población Anticura completando, de esta manera, los resultados inicialmente presentados en Leonardt (2014).

Las definiciones de los distintos tipos de objetos de adorno corporal varían según el autor que se considere, no existiendo un criterio unificado para su clasificación. La mayor parte de los autores diferencia cuentas de pendientes y colgantes empleando como principales criterios de distinción el medio de suspensión, su ubicación y la posible forma de uso (Orchard, 1975; Suárez Diez, 2002; Taborin, 2004; Fernández, 2009). Así, las cuentas han sido definidas como objetos con perforación central (Orchard, 1975; Suárez Diez, 2002; Fernández, 2009) que se habrían empleado de forma agrupada (Suárez Diez, 2002), posiblemente colocadas cara con cara (Fernández, 2009). Asimismo, Taborin (2004) agrega que las cuentas son objetos de pequeño tamaño en relación a los colgantes y pendientes (Taborin, 2004; véase también Della Negra \& Ibáñez Sain Paul, 2012). La diferenciación entre pendientes y colgantes es más compleja aún, ya que en algunos casos se los consideran como un mismo tipo de ornato (por ejemplo Suárez Diez, 2002), mientras que en otros se las definen como tipos distintos (por ejemplo Taborin, 2004). No obstante, en términos generales han sido caracterizados por tener una o más perforaciones (Suárez Diez, 2002), frecuentemente desplazadas respecto del centro (Orchard, 1975; Suárez Diez, 2002; Fernández, 2009), a fin de ser empleados de manera individual (Suárez Diez, 2002; Taborin, 2004; Fernández, 2009). En este trabajo, las cuentas se definen como objetos, generalmente de pequeño tamaño (que no superan los $20 \mathrm{~mm}$ ), con una única perforación que las atraviesa completamente y que suele estar 
centrada, a fin de ser empleadas en conjunto. Los pendientes se definen como objetos de mayor tamaño que las cuentas, de morfología variada y medio de suspensión variado (perforación, muesca o ranura), generalmente desplazado respecto del centro de la pieza.

Cuentas y pendientes se analizaron tecnomorfológicamente a ojo desnudo y con bajos aumentos empleando una lupa binocular Olympus TL (con alcance máximo de 160x) y cámara digital adicionada. Se consideró el tipo de materia prima, la forma y tamaño de la pieza y el tipo y tamaño de la perforación. Según su forma, las piezas se clasificaron en automorfas, categoría que designa a cuentas o pendientes elaborados a partir de valvas de moluscos que conservan la forma biológica original del espécimen empleado (Velázquez Castro \& Juárez Cossío, 2007); cuentas de contorno oval, irregular y circular/subcircular. Dentro de esta última categoría se distinguieron dos tipos que, sólo con fines prácticos, se denominaron cuentas de contorno circular/ subcircular "tipo A" y "tipo B". El primero agrupa a aquellas cuentas cuyos tamaños oscilan entre $6 \mathrm{~mm}$ y $9 \mathrm{~mm}$, mientras que el segundo agrupa a las cuentas que no superan los $2 \mathrm{~mm}$ de diámetro. El tamaño de las piezas se registró considerando sólo los ejemplares enteros. Se tuvo en cuenta, según el caso, diámetro (en las cuentas circulares/subcirculares) o longitud y ancho máximo (en las cuentas ovales, irregulares y automorfas), y el espesor. Las perforaciones se clasificaron en cónicas, bicónicas, uniformes e irregulares (Suárez Diez, 2002) y se tomó el diámetro máximo de las mismas. Todas las medidas fueron registradas en milimetros, empleando un calibre digital. El análisis de los rasgos técnicos consideró la presencia de estrías de abrasión y su localización en la superficie y/o contorno de las cuentas (Velázquez Castro, 2007; Leonardt, 2014, entre otros) y, en el caso de las perforaciones, la presencia de estrías concéntricas en el perfil y/o contorno de las mismas (Velázquez Castro, 2007; Leonardt, 2014, entre otros) o la presencia de microastillamientos ( $\mathrm{d}^{`}$ Errico et al. 2008).

El análisis del conjunto de fragmentos de valva se orientó a la identificación de desechos de manufactura de cuentas. Para ello se consideró la presencia de fragmentos con: a) surcos de corte, b) estrías de abrasión y c) perforaciones, según lo propuesto por Leonardt (2014).

\section{RESULTADOS}

\section{Características de la muestra}

En Población Anticura se recuperaron dos pendientes de valva y 25 cuentas (20 de valva, 4 de roca y una probablemente de valva). Uno de los pendientes y siete cuentas de valva (todas de contorno oval) están fracturadas y 15 presentan evidencias de disolución, lo cual les otorga una textura friable similar al yeso (Gutiérrez Zugasti, 2008-2009). Asimismo, tres cuentas y un pendiente poseen marcas de raíces. Estos tipos de modificaciones tafonómicas también fueron registradas en el conjunto de fragmentos malacológicos, la mayoría de los cuales no supera los $5 \mathrm{~cm}$. Los fragmentos de valva que pudieron ser identificados corresponden, principalmente, a valvas de la especie de agua dulce Diplodon chilensis, que tiene disponibilidad local en el valle del río Manso inferior y, en menor medida, se reconocieron algunos fragmentos correspondientes a valvas de moluscos marinos. Como producto del análisis de este conjunto se identificaron nueve desechos de manufactura de cuentas.

\section{Distribución cronológica y \\ características del conjunto}

Los pendientes proceden exclusivamente de las ocupaciones más tempranas del sitio, correspondientes al Holoceno temprano (8.2007.200 años AP) y medio (ca. 4.700 años AP). Las cuentas y los desechos de manufactura provienen en su totalidad de los niveles datados en el Holoceno tardío (Tabla 1). En los niveles correspondientes al Holoceno tardío inicial (entre 3.350 y 2.270 años AP) se concentra la mayor cantidad de restos óseos humanos (P. Fernández, com. pers. 2014) y se registran cuentas de valva de contorno oval y de contorno circular tipo $\mathrm{A}$, predominando las primeras respecto de las segundas (véase Tabla 1). El conjunto de ocupaciones datadas en momentos del Holoceno tardío final (entre 1.200 y 200 años AP), posee cuentas de contorno oval, de contorno circular (tipo A y B) y de contorno irregular, elaboradas tanto en valva como en roca. A este último lapso se asocian los desechos de manufactura de cuentas de valva registrados en el sitio (véase Tabla 1). 
Tabla 1. Distribución de cuentas, pendientes y desechos de manufactura en relación con los bloques temporales de la secuencia de Población Anticura.

\begin{tabular}{ccccc}
\hline Lapso & Tipo de adorno & Cantidad & Materia prima & $\begin{array}{c}\text { Desechos de } \\
\text { manufactura }\end{array}$ \\
\hline & c. circulares "tipo A" & 6 & valva, roca \\
Holoceno tardío final (200-1200 AP) & c. circulares "tipo B" & 3 & roca, valva (?) \\
& c. ovales & 5 & valva & roca \\
\hline Holoceno tardío inicial (2270-3350 AP) & c. circulares "tipo A" & 1 & valva \\
\hline Holoceno medio (ca. $4700 \mathrm{AP})$ & pendiente automorfo & 2 & valva \\
\hline Holoceno temprano (7200-8200 AP) & pendiente automorfo & 1 & valva \\
\hline Total & & 27 & valva \\
\hline
\end{tabular}

\section{Pendientes}

Los dos pendientes son automorfos y tienen como única modificación antrópica aparente la perforación. El primero de ellos proviene del nivel 23, correspondiente a ocupaciones datadas en alrededor de 8.000 años AP (Bellelli et al. 2013), está fracturado y posee una perforación

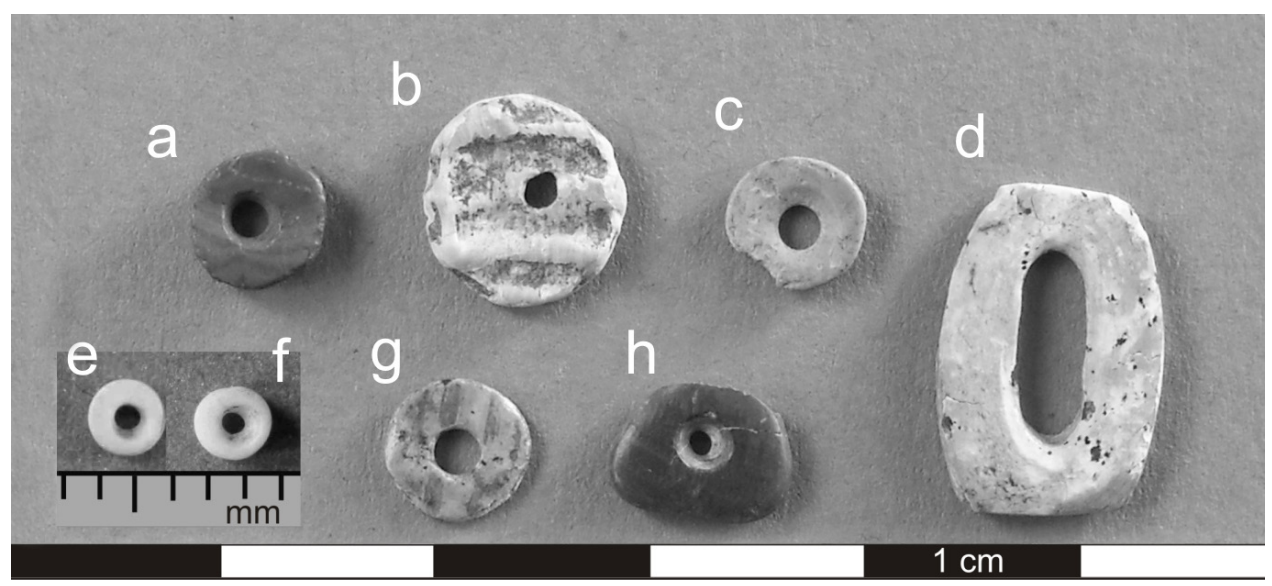

Fig. 1.

Tipos de cuentas y pendientes registrados en Población Anticura:

a-c, g) cuentas circulares "tipo A"; $e-f)$ cuentas circulares "tipo B"; h) cuenta de contorno irregular; d) cuenta de contorno oval; i-j) pendientes.
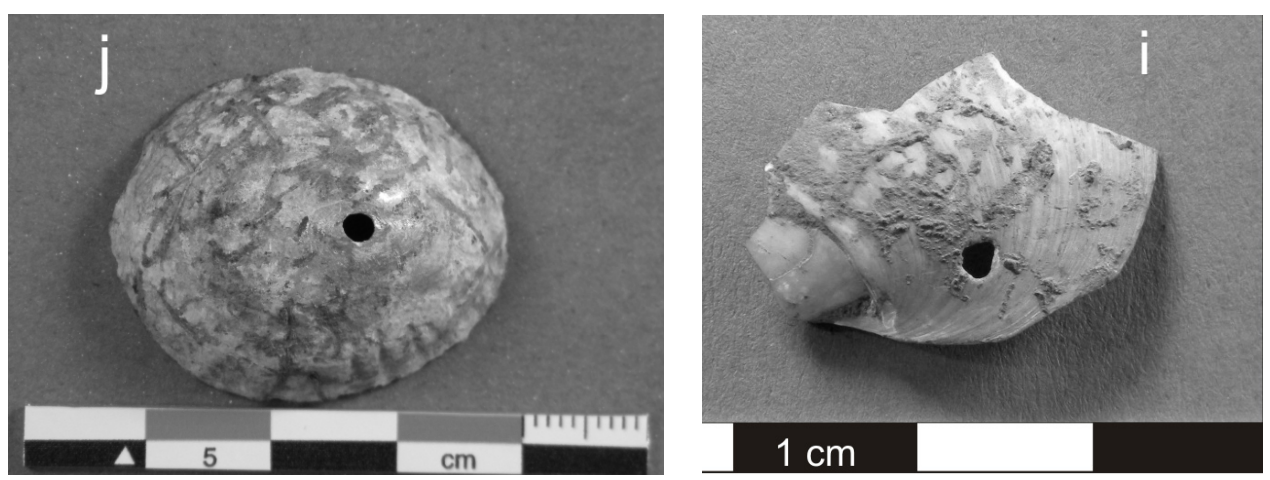
de contorno irregular de $2 \mathrm{~mm}$ de diámetro, realizada cerca del umbo (Fig. 1i). En este caso, las características del umbo permiten atribuirlo, probablemente, a Diplodon chilensis (S. Miquel, com. pers. 2015). El segundo ejemplar está completo y procede de niveles datados en alrededor de 4.700 años AP. Se trata de un ejemplar del molusco marino Nacella sp. de $32 \mathrm{~mm}$ de largo por $26 \mathrm{~mm}$ de ancho que posee una perforación central en el ápice de $2 \mathrm{~mm}$ de diámetro (Fig. 1j). En este caso, aunque la perforación se encuentra centrada y no desplazada respecto del centro de la pieza, su tamaño permitirían asociarlo a un pendiente más que una cuenta. En ambos casos las perforaciones están elaboradas mediante técnica de presión o percusión, lo cual se evidencia por la irregularidad del contorno de la perforación y la presencia de pequeños desprendimientos (Fig. $2 \mathrm{~d}$ ), como producto de la fuerza ejercida con el elemento punzante (d`Errico et al. 2008).

\section{Cuentas}

a. Cuentas de contorno circular "tipo A"

Bajo esta categoría se agrupa un total de ocho cuentas, siete de valva y una lítica. En todos los casos las cuentas de valva presentan caras planas y perforación predominantemente centrada y de tipo cónica. Todas están intensamente formatizadas, por lo que no es posible determinar la especie empleada para su elaboración, excepto una que conserva restos de costillas naturales lo cual permite sugerir que se trata de algún molusco de origen marino, probablemente Aulacomya sp. (Fig. 1b). La cuenta lítica, por su parte, está confeccionada sobre una roca indeterminada de color gris oscuro y presenta una de sus caras planas y la otra levemente cóncava. Su perforación está centrada y es de tipo bicónica (Fig. 1a). Considerando el coeficiente de variación para la variable diámetro de la pieza $(16,5 \%)$ y diámetro de la perforación $(22,6 \%)$ se observa una marcada homogeneidad, independientemente de la materia prima empleada y del bloque temporal considerado (Tabla 2).

En cuanto a las huellas técnicas, en cinco de las piezas (incluyendo la cuenta lítica) se observan estrías concéntricas sobre el perfil de la perforación (Fig. 2a) sugiriendo su realización mediante desgaste por rotación, que implica el desarrollo de movimientos rotatorios con algún tipo de perforador (Suárez Diez, 2002; Velázquez Castro, 2007). En el caso de las cuentas de valva, se ha observado que el empleo de perforadores líticos permite conseguir perforaciones de los diámetros observados y deja, como huella técnica característica, la presencia de estrías profundas y concéntricas tanto en el perfil como en el borde de la perforación (Velázquez Castro, 2007; Leonardt,

Tabla 2. Medidas correspondientes a las cuentas de contorno circular "tipo A".

\begin{tabular}{|c|c|c|c|c|c|}
\hline \multirow{2}{*}{ Lapso } & \multirow{2}{*}{$\begin{array}{l}\mathrm{N}^{\circ} \text { de pieza y } \\
\text { materia prima }\end{array}$} & \multicolumn{2}{|c|}{ Cuenta } & \multicolumn{2}{|c|}{ Perforación } \\
\hline & & Diámetro & Espesor & Diám. máx. & Tipo \\
\hline \multirow{6}{*}{$\begin{array}{l}\text { Holoceno tardío final } \\
\text { (200-1200 años AP) }\end{array}$} & 46 (valva) & 9 & 1 & 2 & cónica \\
\hline & 76 (valva) & 7 & 1 & 2,5 & indeterminada \\
\hline & 11 (valva) & 6 & 1,5 & 1,5 & cónica \\
\hline & 814 (valva) & 6 & 1 & 2 & bicónica \\
\hline & 977 (valva) & 7,5 & 0,5 & 2 & cónica \\
\hline & 913 (roca) & 6 & 1,5 & 1,7 & bicónica \\
\hline \multirow{2}{*}{$\begin{array}{l}\text { Holoceno tardío inicial } \\
\text { (2770-3350 años AP) }\end{array}$} & 130 (valva) & 8 & 1 & 3 & \multirow{2}{*}{$\begin{array}{c}\text { cónica } \\
\text { indeterminada }\end{array}$} \\
\hline & 135 (valva) & 6 & 0,5 & 2,5 & \\
\hline \multicolumn{2}{|c|}{ Promedio } & 6,9 & 1 & 2,2 & \\
\hline \multicolumn{2}{|c|}{ Desvío Estándar } & 1,2 & 0,4 & 0,5 & \\
\hline \multicolumn{2}{|c|}{ Coeficiente de Variación } & $16,5 \%$ & $37,8 \%$ & $22,6 \%$ & \\
\hline
\end{tabular}


2014). En dos de las cuentas de valva también fue posible observar estrías delgadas y paralelas localizadas en el contorno de la pieza y, en un caso, sobre una de sus caras lo cual da cuenta de la regularización de su forma por operaciones de abrasión (véase Leonardt, 2014).

\section{b. Cuentas de contorno circular "tipo B"}

Este grupo está compuesto por tres cuentas de contorno circular de $2 \mathrm{~mm}$ de diámetro cada una y espesor que varía entre 0,5 y $1 \mathrm{~mm}$ (Fig. 1e, f). Dos han sido elaboradas sobre toba vítrea (P. Tchilinguirián com. pers. 2014) mientras que la restante probablemente esté elaborada sobre valva. Presentan perforación centrada de tipo bicónica con diámetro máximo de $1 \mathrm{~mm}$. Las tres cuentas están muy pulidas y no poseen estrías en su contorno o caras. Aunque no se observan estrías técnicas en el perfil de las perforaciones, la presencia de contornos regulares y sección bicónica estaría indicando su elaboración mediante desgaste por rotación. Estas cuentas se recuperaron en microsectores contiguos lo cual permite sugerir que podrian haber conformado parte de un mismo artefacto.

\section{c. Cuentas de contorno oval}

Se trata de 13 cuentas confeccionadas sobre ejemplares de Fissurella sp., obtenidas a partir de la extracción del sector circundante al orificio apical de la valva (Fig. 1d). Todas poseen caras planas y su perforación corresponde al foramen natural del molusco. Al considerar las medidas de las cuentas enteras $(n=6)$ de ambos bloques temporales (Tabla 3 ) se observa una marcada similitud en el tamaño de las piezas y del orificio, lo cual estaría indicando una selección en la talla de los individuos empleados para elaborarlas. En cuanto a las huellas técnicas, cuatro de estas piezas presentan estrías de abrasión en una de sus caras (Fig. 2e) y cuatro también en el contorno, lo que sugiere que su forma fue regularizada mediante abrasión. Asimismo, tres de ellas, dos procedentes del lapso 3.350-2.270 años AP ( $\mathrm{N}^{\circ} 129$ y 1.010) y otra del lapso 1.200-200 años AP ( $\mathrm{N}^{\circ}$ 951) poseen restos de pigmento rojo adherido.

\section{d. Cuentas de contorno irregular}

Se halló una única cuenta de contorno irregular cuya materia prima es una roca indeterminada de color gris oscuro (Fig. 1h). Esta pieza presenta ambas caras planas y sus medidas máximas son $8 \mathrm{~mm}$ por $6 \mathrm{~mm}$ y espesor de $1 \mathrm{~mm}$. La perforación es de tipo bicónica con un diámetro máximo de $1,5 \mathrm{~mm}$ y posee estrías concéntricas en el perfil de dicha perforación que dan cuenta de su realización mediante desgaste por rotación. Esta pieza tiene un sector del borde fracturado.

Fragmentos de valva

Del total de 630 fragmentos de valva, sólo nueve pudieron ser identificados como desechos de manufactura de cuentas. Se trata, en su

Tabla 3. Medidas correspondientes a las cuentas de contorno oval enteras.

\begin{tabular}{ccccccc}
\hline \multirow{2}{*}{ Lapso } & \multirow{2}{*}{$\mathrm{N}^{\circ}$ de pieza } & Largo & Ancho & Espesor & Largo & Ancho \\
\hline Holoceno tardío final & 105 & 13 & 10 & 1 & 8 & 3,5 \\
$(200-1.200$ años AP) & 951 & 13 & 10 & 1 & 9 & 4 \\
\hline \multirow{2}{*}{ Holoceno tardío inicial } & 129 & 15 & 10 & 1 & 8 & 3 \\
$(2.270-3.350$ años AP) & 1.077 & 13 & 9 & 1 & 6 & 3 \\
& 1.009 & 14 & 10 & 1 & 8,5 & 4 \\
\hline Promedio & 1.010 & 14 & 12 & 1 & 9 & 4 \\
\hline Desvío Estándar & & 13,7 & 10,2 & 1 & 8,1 & 3,6 \\
\hline Coeficiente de Variación & & 0,8 & 0,9 & & 1,1 & 0,5 \\
\hline \multicolumn{2}{c}{} & $6 \%$ & $9,7 \%$ & & $13,8 \%$ & $13,7 \%$ \\
\hline
\end{tabular}



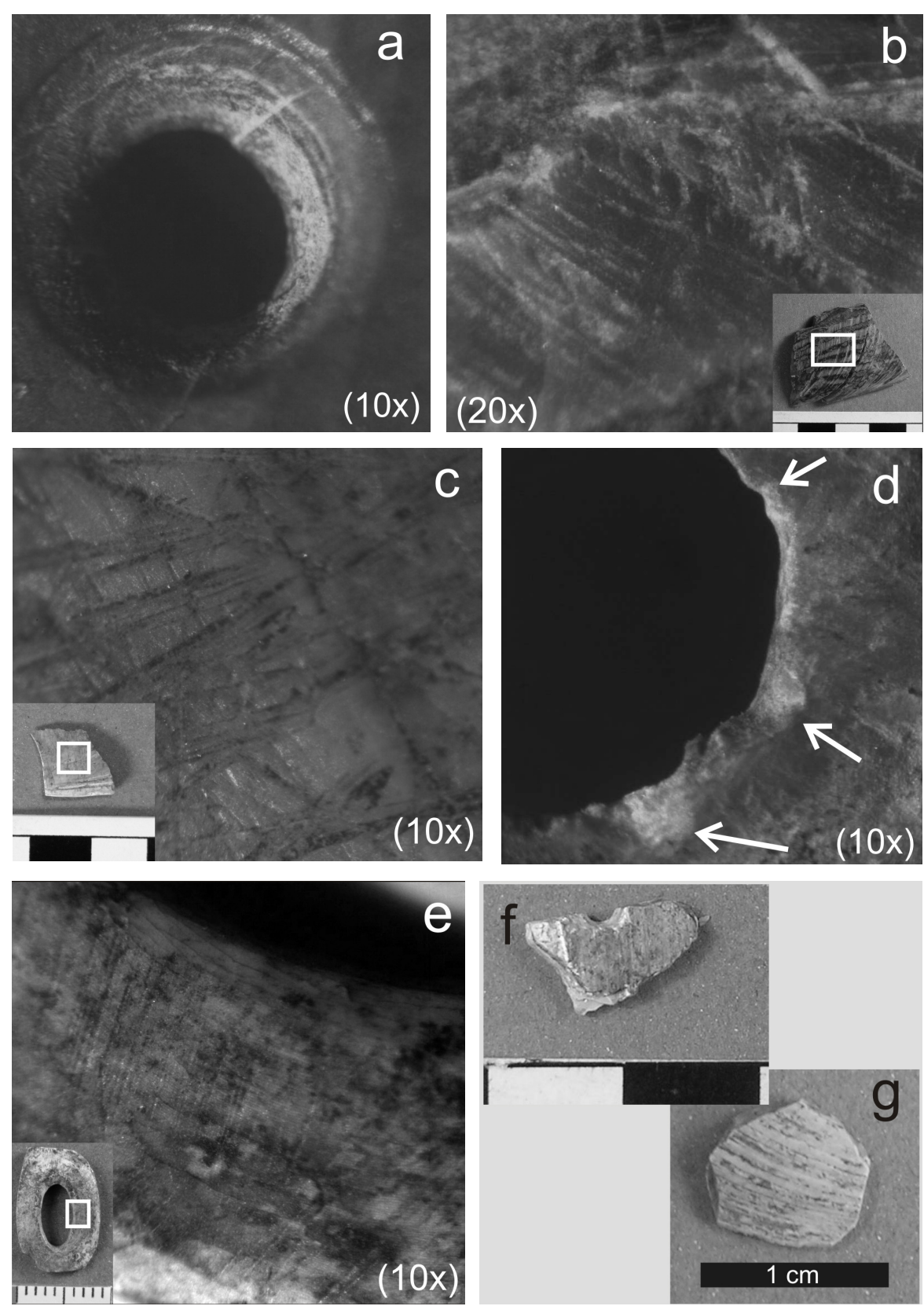

Fig. 2. Huellas de manufactura registradas en la muestra de Población Anticura: a) detalle de estrías de perforación en cuenta lítica; $b, c)$ detalle de estrías de abrasión en fragmentos de valva; d) detalle de microdesprendimientos (señalados por flechas blancas) en perforación de pendiente de valva; e) detalle de estrías de abrasión en superficie de cuenta oval; f) fragmento de valva fracturado con perforación; g) preforma de cuenta.

mayoría ( $\mathrm{n}=6)$ de trozos de valva con estrías de abrasión en una de sus caras (Figs. 2 b, c). Uno de ellos corresponde a un posible fragmento de Diplodon chilensis (Leonardt, 2014) mientras que en dos casos, las estrías se ubican sobre fragmentos que presentan restos de costillas naturales, probablemente perteneciente al molusco marino Aulacomya sp. (Fig. 2 b). Para el resto de los desechos identificados, si bien no se pudo determinar la especie de molusco empleado, su coloración $y / 0$ espesor permitiría sugerir que también se trata de moluscos de origen marino. Un fragmento de valva presenta un surco de corte de sección en $V$ que atraviesa su superficie y en cuyos perfiles se disponen estrías longitudinales. No obstante, no se observaron huellas de corte en el contorno del fragmento como sería esperable de ser producto de la extracción de formas base 
para la elaboración de cuentas (véase Leonardt, 2014). Otro corresponde a un fragmento de contorno irregular que presenta una perforación que está fracturada, de $3 \mathrm{~mm}$ de diámetro máximo y sección cónica (Fig. 2f). Aunque no se pudieron identificar estrías en el perfil de esta perforación, ya que presenta un importante grado de exfoliación, la forma de su sección, la regularidad del contorno del orificio y su diámetro concuerdan con el patrón esperable para una perforación antrópica (véase Leonardt, 2014). Por último, se identificó un trozo de valva de contorno subcircular con perfiles de fractura y diámetro de $9 \mathrm{~mm}$ que se corresponde con el esperable para una preforma de cuenta regularizada mediante técnica de presión o percusión (Fig. 2 g, véase Laporte et al. 1994).

Estos desechos, considerados en conjunto, dan cuenta de las distintas etapas de manufactura de cuentas (véase Leonardt, 2014: Fig. 4). Todos se distribuyen entre los niveles correspondientes al conjunto de ocupaciones datadas en el Holoceno tardío final (entre 1.200 y 200 años AP). En estos niveles se concentra el $82 \%(n=505)$ del total de fragmentos recuperados en el sitio así como dos charnelas de mitílidos que, aunque no evidencian huellas de trabajo podrían ser resultado de la primera etapa de manufactura de cuentas (véase Leonardt, 2014).

Finalmente, cabe destacar que una alta proporción de fragmentos presentó evidencias de disolución $(18 \%, n=113)$ y de exfoliación $(32 \%$, $\mathrm{n}=201$ ), lo que da cuenta de la degradación de las valvas e impide la identificación de huellas de manufactura. Esta situación, sumada a la información generada experimentalmente, que permite sostener que el proceso de elaboración de cuentas de valva genera poca cantidad de fragmentos con huellas de manufactura plausibles de ser identificadas arqueológicamente (véase Leonardt, 2014), podría estar explicando, al menos en parte, la baja cantidad de desechos de manufactura identificados en la muestra.

\section{CUENTAS Y PENDIENTES A ESCALA REGIONAL EN PATAGONIA CONTINENTAL}

En la Fig. 3 se presenta la localización aproximada de los sitios arqueológicos registrados en la bibliografía de Patagonia en los que se

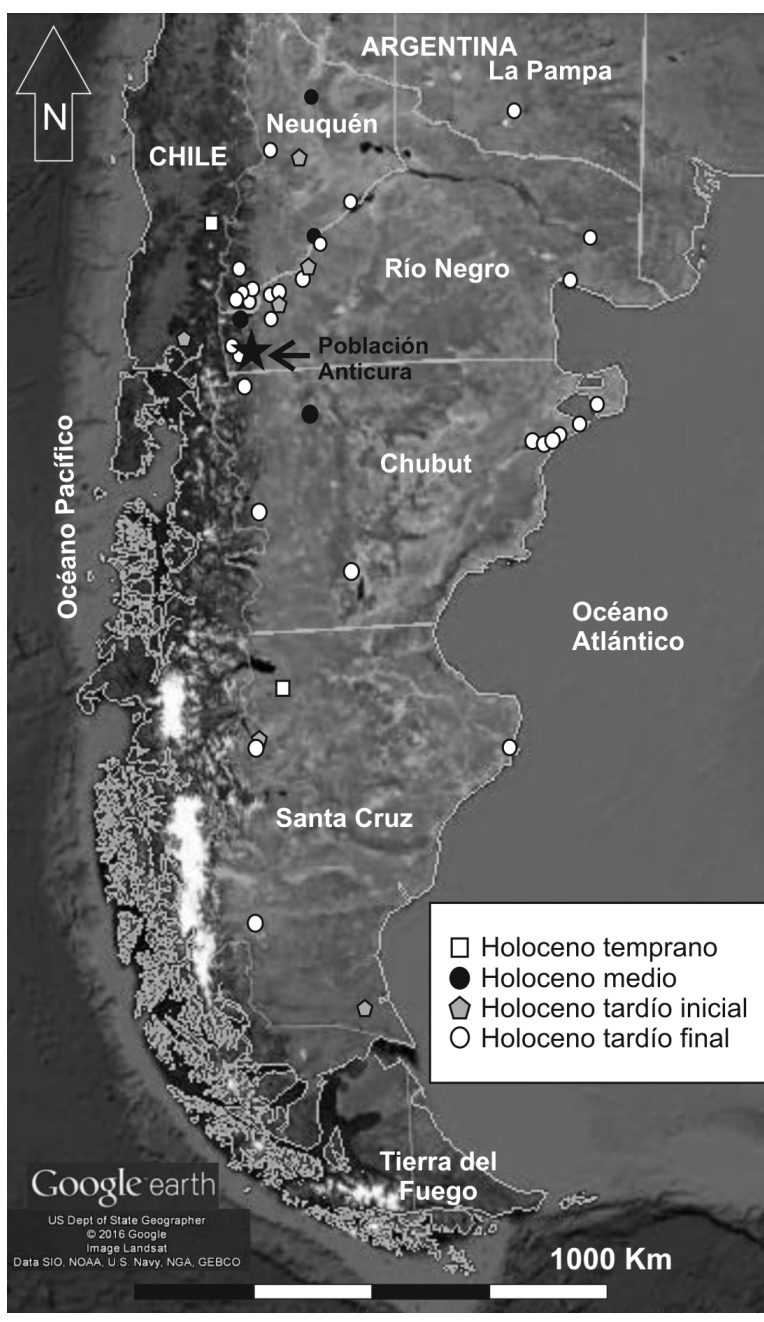

Fig. 3. Distribución aproximada de sitios arqueológicos con cuentas y pendientes registrados en la bibliografía de Patagonia continental.

menciona la presencia de cuentas y pendientes. De 48 contextos con asociaciones cronológicas publicadas, sólo seis (12\%) están datados en el Holoceno temprano y medio (Fig. 4a). En el primer caso se trata de una cuenta oval de valva procedente de los niveles inferiores de Cueva de las Manos (Gradin et al. 1976) y un pendiente de hueso en el sitio Alero Marifilo 1, en Chile (Adán et al. 2004). Para el Holoceno medio se mencionan tres pendientes y cuentas elaboradas con pequeñas valvas de Homalopoma sp. en el sitio El Trébol (Hajduk et al. 2008). También, cuentas de valva en Piedra de Águila 11(Sanguinetti de Bórmida \& Curzio, 1996), Campo Moncada 2 (Bellelli, 1983) y 
Aquihuecó, este último, un sitio enterratorio (Della Negra \& Ibañez Saint Paul, 2012). Finalmente, la mayor parte de los contextos publicados corresponden a momentos datados en el Holoceno tardío, particularmente a los últimos 2.000 años.

$\mathrm{Si}$ bien cuentas y pendientes se registran a lo largo de todo el Holoceno y, en varios casos, en los mismos contextos (véase por ejemplo Hajduk et al. 2008; Fernández \& Ramos, 2009; Della Negra \& Ibáñez Sain Paul, 2012), durante momentos del Holoceno tardío final (1.900-200 años $\mathrm{AP}$ ) las menciones de cuentas respecto de pendientes se incrementan notablemente (Fig. 4b). En cuanto a las materias primas empleadas para su elaboración, las más representadas son valva, roca y hueso. En 39 de los 48 contextos se registra el uso de valva, evidenciando continuidad desde momentos del Holoceno temprano $y$ un predominio en los contextos datados en el Holoceno tardío final (Fig. 5a). Cabe destacar que en 23 de estos 39 contextos las menciones sólo refieren al empleo de valva como materia prima, mientras que en los 16 restantes los adornos de valva se registran junto con adornos de hueso, roca o ambas (Fig. 5a). Sólo en menor medida se menciona el empleo otras materias primas tales como cerámica, madera y cáscara de huevo, todas correspondientes a contextos datados en el Holoceno tardío final (véase

b

$$
\begin{aligned}
& \text { sólo cuentas } \\
& \text { sólo pendientes } \\
& \text { cuentas y pendientes }
\end{aligned}
$$

por ejemplo Sanguinetti de Bórmida \& Curzio, 1996; Cimino, 2007; Crivelli Montero \& Ramos, 2009). Por último, en cuanto a las morfologías mencionadas, en el caso de los pendientes éstas son variadas (automorfo, alargado, irregular, trapezoidal, etc.) en los distintos contextos considerados (véase por ejemplo Fernández, 2009; Fernández \& Ramos, 2009; Della Negra \& Ibañez Sain Paul, 2012) mientras que, en el caso de las cuentas, si bien también se registra una considerable variabilidad morfológica, para momentos del Holoceno tardío final las más frecuentemente mencionadas son las cuentas de morfología circular/subcircular (Fig. 5b).
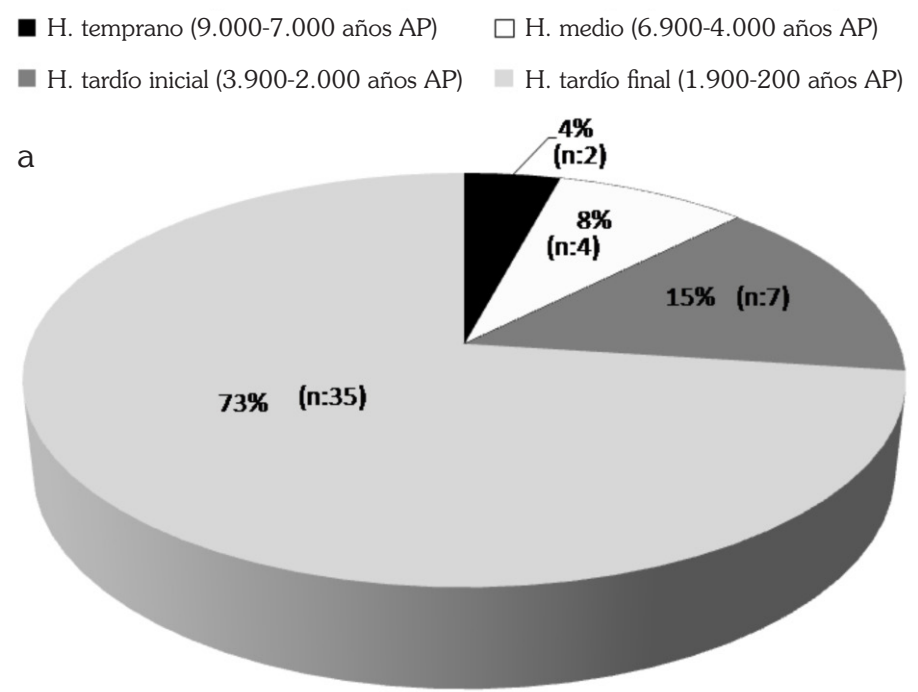

30

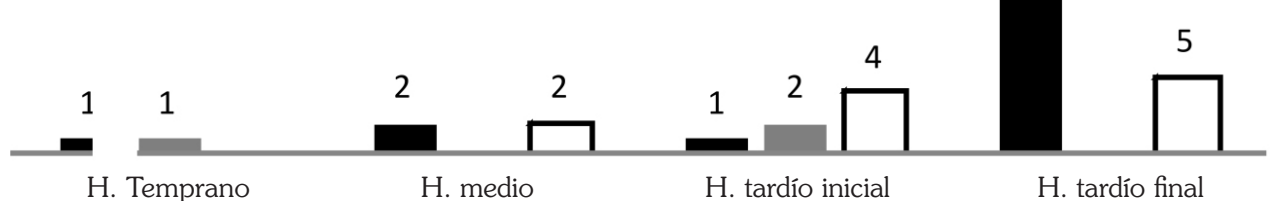

Fig. 4. a) Proporción de contextos con presencia de cuentas y pendientes según distintos momentos del Holoceno ( $\mathrm{N}$ : 48 contextos); b) frecuencias de menciones de cuentas y pendientes en los distintos contextos publicados para el Holoceno ( $\mathrm{N}: 48$ contextos). 
a
sólo valva
$\boldsymbol{\nabla}$ valva y hueso
$\mathbf{X}$ valvaa, roca y otro
E valva, hueso, roca y otro

sólo hueso

$\boldsymbol{\nabla}$ valva, hueso y otro

hueso y roca

III $\sin$ dato
■ sólo roca

\ valva y roca

II valva, hueso y roca

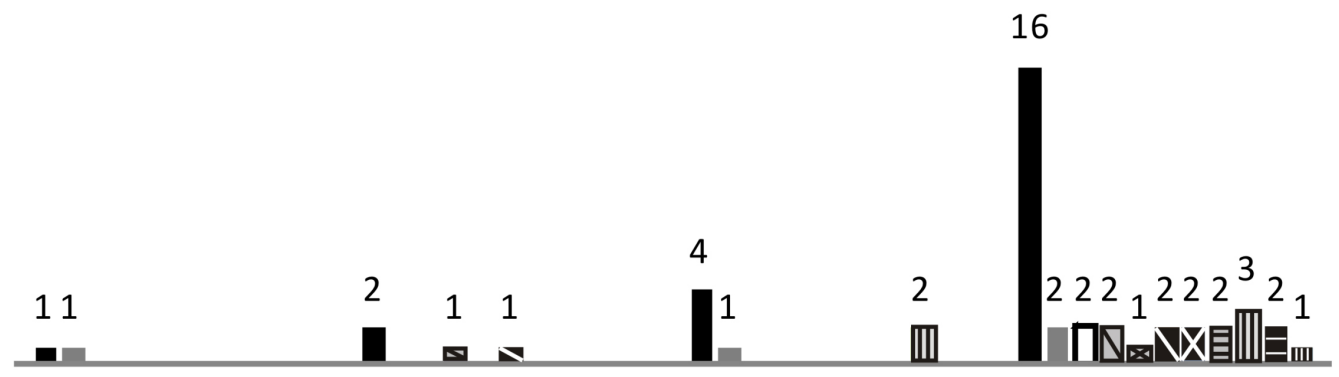
H. Temprano
H. medio
H. tardío inicial
H. tardío final

b

- sólo circular/subcircular
circular/subcircular y otras formas
口 otras formas
sin dato

\author{
16
}

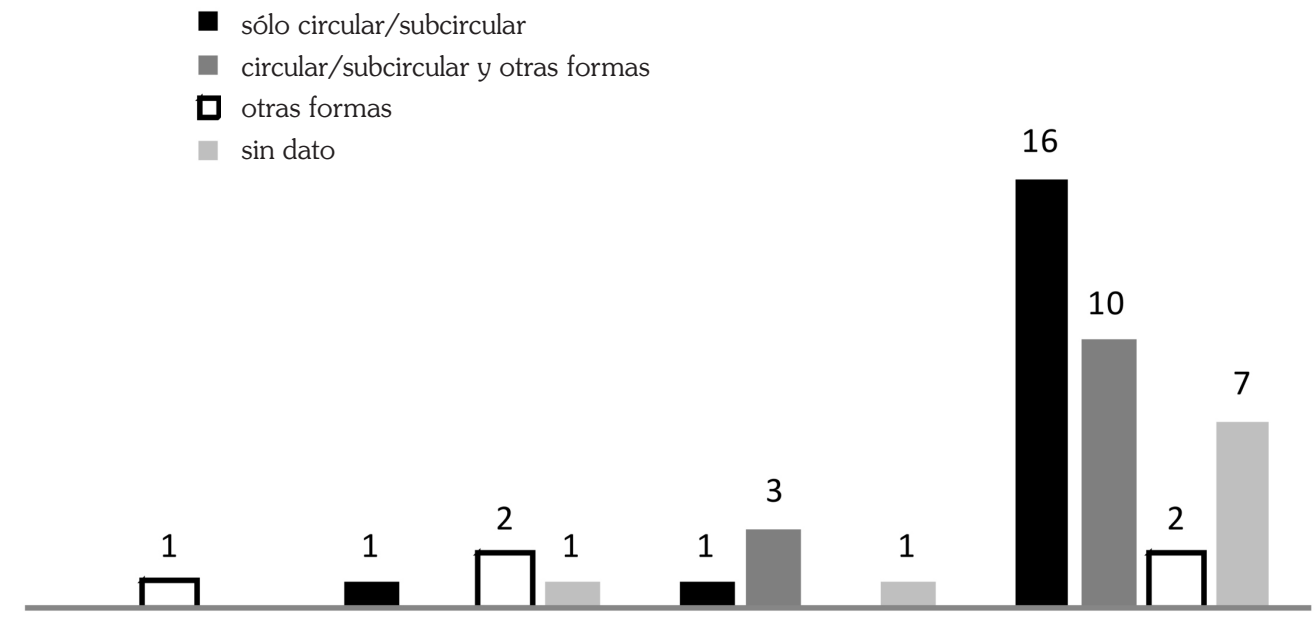

H. Temprano

H. medio

H. tardío inicial

H. tardío final

Fig. 5. a) frecuencias de menciones de materias primas en los distintos contextos publicados para el Holoceno; b frecuencias de menciones morfologías en los distintos contextos publicados para el Holoceno.

\section{DISCUSIÓN}

A partir del análisis realizado, es posible plantear algunas tendencias generales. En primer lugar, si bien los artefactos de adorno personal están presentes a lo largo de toda la secuencia temporal de Población Anticura, sus frecuencias varían notablemente entre las ocupaciones más tempranas y las correspondientes al Holoceno tardío (3.300200 años AP). En el primer caso, las evidencias se restringen únicamente a dos pendientes de valva mientras que, en el segundo caso, la frecuencia de artefactos de adorno personal aumenta y está representada únicamente por cuentas. Asimismo, para los últimos 1.200 años, en correspondencia con el incremento general de los restos materiales recuperados en el sitio (Fernández et al. 2013), se registra la mayor diversidad de cuentas y las evidencias de producción local de cuentas de valva.

Tanto las cuentas ovales como las circulares 
muestran una marcada similitud en los tamaños independientemente del bloque temporal en el que se recuperaron. En las primeras, esta similitud refleja la búsqueda de moluscos de la misma talla, ya que su morfología está dada principalmente por las características naturales de las valvas de Fissurella sp. La mayor parte de este conjunto está asociada a los eventos mortuorios correspondientes al lapso 3.350-2.270 años AP. Incluso dos de las cuentas halladas en estos niveles poseen restos de pigmento rojo adherido, característica que también fue registrada en parte de los restos óseos humanos (Fernández et al. 2013). No obstante, por el momento su presencia en el sitio no puede asociarse únicamente a estos eventos mortuorios ya que otra parte de este conjunto de cuentas procede de los niveles correspondientes al bloque temporal 1.200-200 años AP. En este sentido, el registro de galerías de roedores en estos niveles y la presencia de marcas de raíces en algunas de las cuentas de contorno rectangular permitiría sugerir la posibilidad de migración vertical de parte de las piezas. Situaciones similares están siendo registradas en otros materiales recuperados en el sitio y actualmente están bajo análisis (P. Fernández, com. pers. 2014).

En el caso de las cuentas de contorno circular todas, excepto dos, se concentran en las ocupaciones correspondientes a los últimos 1.200 años y la similitud observada tanto en las piezas elaboradas en valva como en roca sugiere la búsqueda de un patrón morfológico particular, independientemente del material soporte. Esta situación tiene correspondencia con lo observado en otros sitios del valle del río Manso inferior donde se han hallado cuentas circulares elaboradas en distintas materias primas. Así, en el sitio Paredón Lanfré se recuperó una cuenta circular de valva y otra modelada en arcilla (véase Fernández et al. 2011) de tamaño similar a las cuentas circulares de tipo A mencionadas en este trabajo. Además, en este sitio y en otro denominado Campamento Argentino se recuperaron cuentas similares a las tipo B, en contextos datados entre 560 y 200 años AP (Ciarlo et al. 2010).

En cuanto a la elaboración de estos artefactos, la presencia de desechos de manufactura de cuentas de valva en el lapso de ocupaciones más tardías, junto con el registro de artefactos aptos para elaborarlas tales como perforadores líticos y manos y molinos (Leonardt, 2014), permiten sugerir que al menos parte de las cuentas circulares de valva podrían haberse producido en el sitio. Por el contrario, la ausencia de restos de valvas de Fissurella sp. permite hipotetizar su ingreso como objetos terminados. En el caso de las cuentas líticas, la posibilidad de su producción local queda abierta a partir de la disponibilidad de toba volcánica en el área (Carballido Calatayud \& Pereyra, 2012). Cabe destacar que las cuentas líticas registradas en Campamento Argentino y Paredón Lanfré también se elaboraron en rocas disponibles en las cercanías (filitas), aspecto que llevó a los autores a sugerir su producción local (Ciarlo et al. 2010). Además, la identificación de los mismos patrones de huellas en las perforaciones que las cuentas de valva permite inferir el empleo de técnicas similares de manufactura. De hecho, trabajos experimentales han probado la eficacia del empleo de perforadores líticos para trabajar tanto valva como hueso y roca (Gurova et al. 2013).

El predominio de la valva como materia prima para la elaboración de adornos corporales es evidente a lo largo de toda la secuencia ocupacional del sitio y el único registrado para los pendientes de momentos tempranos. En este sentido, si bien en el caso de las cuentas líticas es posible sostener un aprovechamiento de materias primas locales, la situación es diferente respecto de las valvas de moluscos, donde se observa una tendencia a la selección de especies marinas. Esto se evidencia tanto en los desechos de manufactura (la mayoría corresponden a valvas de moluscos marinos) como en las características de parte de las cuentas y pendientes. Al respecto, Nacella sp. (especie identificada en el pendiente datado en Holoceno medio), Fissurella sp. y Aulacomya sp. (ambas identificadas para momentos tardíos) tienen una distribución geográfica que abarca tanto el océano Atlántico como el Pacífico, desde península Valdés hasta la isla de Chiloé (Forcelli, 2000). Así, considerando que Población Anticura se localiza a aproximadamente $60 \mathrm{~km}$ lineales del océano Pacífico y que existen evidencias arqueológicas en el área que permiten sostener la interacción y/o circulación de cazadores-recolectores terrestres a ambos lados de la cordillera (Bellelli et al. 2008), se podría especular que la costa pacífica podría 
haber funcionado como área de obtención de los moluscos. No obstante, hasta el momento no se han registrado otras evidencias que indiquen alguna relación con los pueblos costeros localizados en la vertiente occidental de los Andes (Bellelli et al. 2008). Por otro lado, las características del registro material y del arte rupestre permiten integrar a Población Anticura en las redes de comunicación $y$ los procesos sociales registrados en el sector occidental de los Andes para momentos tardíos (Bellelli et al. 2008), por lo cual la costa atlántica también podría haber funcionado como fuente de obtención de estos moluscos. En este sentido, cabe destacar que Silveira y colaboradores (2010) mencionan la presencia de un fragmento de valva de Adelomelon brasiliana en el sitio Alero Los Cipreses, en el área boscosa de lago Traful (Argentina), distante a $500 \mathrm{~km}$ de la costa atlántica, área de distribución de este molusco.

Comparando las tendencias observadas en Población Anticura con los patrones registrados a partir de la información publicada en Patagonia continental es posible establecer ciertas correspondencias. En primer lugar, la escasa representación de artefactos de adorno personal evidenciada en las ocupaciones tempranas del sitio concuerda con la situación observada a escala regional. En la mayor parte de los casos se trata del hallazgo de una o dos piezas en contextos no mortuorios (Gradin et al. 1976; Sanguinetti de Bórmida \& Curzio, 1996; Hajduk et al. 2004). Asimismo, el empleo de valvas de moluscos como materia prima para la elaboración de estos artefactos se registra en otros sitios arqueológicos desde momentos tempranos, como en Cueva de las Manos (Gradin et al. 1976) y El Trébol (Hajduk et al. 2008). Sin embargo, no es la única materia prima registrada así como tampoco la presencia de pendientes se restringe a momentos tempranos (véase por ejemplo Hajduk et al. 2008; Della Negra \& Ibáñez Saint Paul, 2012). La presencia de los dos pendientes registrados en Población Anticura constituye un nuevo aporte a la variabilidad que se observa en el registro de artefactos de adorno de momentos del Holoceno temprano y medio en la región. Esto puede sostenerse en base a que, si bien la muestra regional de estos contextos es reducida en relación a la de momentos tardíos
(Holoceno tardío inicial y final), en ambas se observan los mismos tipos de ornatos (cuentas y pendientes), el empleo de las mismas materias primas (predominio de valva, hueso y roca) y una considerable variabilidad morfológica, lo cual no estaría determinado por el tamaño de la muestra.

$\mathrm{Al}$ contrario, la información regional disponible para contextos tardíos permite sugerir la tendencia hacia una mayor homogeneidad en la producción de artefactos de adorno, manifestada en el predominio de las cuentas respecto de los pendientes. Asimismo, el dominio de las formas circulares/subcirculares, elaboradas en distintos tipos de soportes (entre ellos valva, roca y hueso) que prevalece en los contextos tardíos se refleja a escala de sitio en población Anticura. Asimismo, las menciones de cuentas líticas se restringen, en su totalidad, a contextos datados con posterioridad a los últimos 2.000 años (Onetto, 1986-1987; Silveira, 1996; Gómez Otero, 2007; Prates, 2008; Crivelli Montero \& Ramos, 2009; Fernández, 2009; Ciarlo et al. 2010), lo cual podría sugerir que la incorporación de este material para la elaboración de cuentas sería una selección de momentos tardíos. Otro aspecto que encuentra correspondencia con la información regional son las evidencias de producción local de artefactos de adorno. Los casos en los que se mencionan contextos de elaboración local de cuentas corresponden a contextos datados en los últimos 2.000 años y comparten las características observadas en el sitio: escasa cantidad de desechos de manufactura y/o piezas semiformatizadas, en algunos casos asociada a perforadores líticos (véase por ejemplo Cimino, 2007; Fernández \& Ramos, 2009). Esta situación responde, en principio, a las características propias de la cadena operativa de elaboración de cuentas en contextos de producción no especializada (Leonardt, 2014) pero también puede estar relacionada con la escases de análisis que apunten a identificar este tipo de contextos. $\mathrm{Al}$ respecto, cabe destacar que si bien en varios casos se menciona el empleo de valvas marinas (por ejemplo Silveira, 1996; Fernández \& Ramos, 2009), las valvas de moluscos de agua dulce también fueron aprovechadas para la elaboración de cuentas y pendientes (Cassiodoro, 2005; Prates, 2008; Fernández \& Ramos, 2009; Leonardt, 2014). 


\section{CONSIDERACIONES FINALES}

Este análisis es un primer paso para comenzar a conocer las características de la producción, uso y distribución de cuentas en Patagonia continental y evaluar el papel que pudieron haber tenido en el contexto regional de momentos tardíos. En principio, tanto la información a escala de sitio obtenida en Población Anticura como la registrada a escala regional en Patagonia continental se corresponden con las expectativas planteadas. Si bien cuentas $y$ pendientes se registran desde momentos tempranos, se pueden observar variaciones en su producción a lo largo del Holoceno. Por un lado, durante el Holoceno temprano y medio el registro de estos artefactos evidencia una considerable variabilidad en formas y materias primas. No obstante, su presencia no deja de ser llamativa, sobre todo teniendo en cuenta que mayormente los hallazgos correspondientes a ocupaciones tempranas se componen de escasas evidencias arqueológicas. Esta situación, es sugerente en cuanto al rol que pudieron haber tenido estos artefactos en la vida cotidiana desde estos momentos. Además, la presencia de valva como materia prima para su elaboración sugiere algún tipo de carácter simbólico otorgado a estos materiales (por ejemplo Bonomo, 2007).

Por otro lado, el predominio de cuentas sobre pendientes y posiblemente la recurrencia de un tipo de morfología específica para momentos tardíos están en correspondencia con un escenario como el propuesto donde la existencia de una morfología particular podría estar dando cuenta de elecciones tecnológicas concretas en relación con patrones de diseño compartidos. En este sentido, tal como sostienen Kuhn y Stiner (2007), en términos de comunicación visual, el éxito de la transmisión de un mensaje en parte es una función de la redundancia de su representación. Si bien la valva juega un papel dominante, la representación de la misma morfología en distintas materias primas sugiere que la selección de la forma prima por sobre el tipo de material empleado. Por último, las evidencias de producción local en distintos sectores de Patagonia (Fernández, 2009; Prates, 2008; Fernández \& Ramos, 2009; Leonardt, 2014) permiten sostener la hipótesis de una producción no localizada para momentos tardíos lo cual, en principio, se corresponde con la idea de replicabilidad de un código. No obstante, es necesario profundizar en el análisis de este registro que hasta ahora ha recibido poca atención en la arqueología regional. En este sentido, la incorporación del análisis de conjuntos de artefactos de adorno corporal de distintos sectores de Patagonia, considerando la existencia de estandarización al interior y entre los conjuntos, selección de materias primas, características tecnológicas y contextos temporales y de hallazgo permitirá evaluar en mayor profundidad estas hipótesis. Estas tareas se están efectuando actualmente en conjuntos arqueológicos procedentes de las provincias de Río Negro, Chubut y Santa Cruz. A su turno, la identificación de otros contextos de producción local de cuentas y la incorporación del análisis de la producción de cuentas líticas permitirá profundizar respecto de las características de estos artefactos en Patagonia y la variabilidad de las elecciones técnicas desarrolladas. Finalmente, resta también evaluar la incidencia de los distintos factores que podrían estar sesgando el registro arqueológico de artefactos de adorno, tales como problemas de preservación, características de los contextos de recuperación, huaqueo y disparidad de información publicada, a fin de refinar la información respecto de estos artefactos a escala regional.

\section{AGRADECIEMIENTOS}

Quiero agradecer a Vivian Scheinsohn, Pablo Fernández e Isabel Cruz por sus valiosos aportes directos e indirectos a este trabajo. Al Dr. Pablo Tchilinguirián (INAPL) por su amable observación de algunas de las cuentas líticas, al Dr. Sergio Miquel (MACN) por la identificación de uno de los pendientes analizados y a los miembros del equipo de la $\mathrm{CA} 42^{\circ}$. Finalmente quiero agradecer al Consejo Nacional de Investigaciones Científicas y Técnicas (CONICET) que financia esta investigación a través de una beca de doctorado.

\section{BIBLIOGRAFÍA}

Adán, L., Mera, R., Becerra, M., \& Godoy, M. (2004). Ocupación arcaica en territorios boscosos y lacustres de la región precordillerana andina del centro-sur de Chile. 
El sitio Marifilo-1 de la localidad de Pucura. Chungará, volumen especial, 1121-1136.

Barton, M. C., Clark, G. A., \& Cohen, A. E. (1994). Art as information: explaining Upper Palaeolithic Art in western Europe. World Archaeology, 26(2), 185207.

Belardi, J. B. (2004). Más vueltas que una greca. En M. T. Civalero, P. M. Fernández, \& A. G. Guraieb (Eds.), Contra viento y marea. Arqueología de Patagonia (pp. 591-603). Buenos Aires: Instituto Nacional de Antropología y Pensamiento LatinoamericanoSociedad Argentina de Antropología.

Bellelli. C. T. (1983). Sitio Campo Moncada 2. En Arqueologia del Chubut. El valle de Piedra Parada (pp. 31-42). Rawson: Dirección Provincial de Cultura del Chubut.

Bellelli, C., Carballido Calatayud, M., \& Fernández, P. M. (2013). Ocupaciones tempranas del bosque Norpatagónico: el sitio Población Anticura (valle del río Manso inferior, Río Negro). En J. R. Bárcena, \& S. E. Martín, (Eds.), Arqueología Argentina en el Bicentenario de la Asamblea General Constituyente de 1813 (p. 516). La Rioja: Universidad Nacional de La Rioja.

Bellelli, C., Scheinsohn, V., \& Podestá, M. M. (2008). Arqueología de pasos cordilleranos: un caso de análisis en la Comarca Andina del Paralelo $42^{\circ} \mathrm{y}$ áreas vecinas durante el Holoceno Tardío. Boletín del Museo Chileno de Arte Precolombino, 13(2), 37-55.

Bonomo, M. (2007). El uso de los moluscos marinos por los cazadores-recolectores pampeanos. Chungará, 39(1), 87-102.

Borrero, L. A. (1994-1995). Arqueología de la Patagonia. Palimpsesto, 4, 9-69.

Borrero, L. A. (2001). El poblamiento de la Patagonia: toldos, milodones y volcanes. Buenos Aires: Emecé.

Boschín, M. T. (2009). Tierra de Hechiceros: arte indígena de Patagonia Septentrional Argentina. Córdoba: Universidad de Salamanca - Universidad de Córdoba.

Carballido Calatayud, M., \& Pereyra, F. X. (2012). Determinación de la base regional de recursos líticos del área río Manso inferior-Foyel (Río Negro). Primeros resultados. Comechingonia, 16, 287-296.

Cassiodoro, G. (2005). Tecnología malacológica de los entierros humanos del lago Salitroso (Santa Cruz, Argentina). Relaciones de la Sociedad Argentina de Antropología, 30, 257-262.

Cassiodoro, G., \& García Guraieb, S. (2009). Análisis del registro tecnológico y osteológico de los entierros humanos del Holoceno tardío del lago Salitroso
(Santa Cruz): un aporte al estudio del comportamiento mortuorio de cazadores-recolectores. En M. Salemme, F. Santiago, M. Álvarez, E. Piana, M. Vázquez, \& M. E. Mansur (Eds.), Arqueología de la Patagonia. Una mirada desde el último confín (pp. 613-628). Ushuaia: Utopías.

Ciarlo, N. C., Solá, P., \& Bellelli, C. (2010). Caracterización de cuentas líticas provenientes del valle del río Manso (provincia de Río Negro). En S. Bertolino, R. Cattaneo, \& A. Izeta (Eds.), La Arqueometría en Argentina y Latinoamérica (pp. 159-164). Córdoba: Universidad Nacional de Córdoba.

Cimino, A. (2007). Arqueomalacología en las sierras de la vida: análisis de los adornos realizados sobre materia prima malacológica hallados en el sitio Chenque I (P.N.L.C., provincia de La Pampa). En C. Bayón, A. Pupio, M. I. González, N. Flegenheimer \& M. Frère (Eds.), Arqueología de las Pampas Tomo I (pp. 309-324). Buenos Aires: Sociedad Argentina de Antropología.

Crivelli Montero, E. A., \& Ramos, M. S. (2009). Hallazgos especiales de Rincón Chico 2/87. En E. A. Crivelli Montero, M. M. Fernández \& M. S. Ramos (Eds.), Arqueología de rescate en Rincón Chico, provincia del Neuquén (pp. 205-213). Buenos Aires: Editorial Dunken.

d`Errico, F., Vanhaeren, M., \& Wadley, L. (2008). Possible shell beads from the Middle Stone Age layers of Sibudu Cave, South Africa. Journal of Archaeological Science, 35, 2675-2685.

Della Negra, C. E., \& Novellino, P. (2002). Nuevos estudios sobre los antiguos habitantes de la cuenca del río Limay: Sitio Grande, departamento Picun Leufu, provincia del Neuquén. Relaciones de la Sociedad Argentina de Antropología, 27, 101-113.

Della Negra, C. E., \& Ibañez Saint Paul, V. (2012). Adornos personales durante el Holoceno en Neuquén, su relevancia simbólica. Comechingonia Virtual, 6(1), 39-58.

Fernández, M. (2009). Los adornos personales en el noroeste patagónico: Contexto y cronología. En E. Cordeu (Ed.), VI Congreso Americanistas Tomo II (pp. 125-149). Buenos Aires: Sociedad Argentina de Americanistas.

Fernández, M., \& Ramos, M. (2009). Hallazgos especiales del sitio Casa de Piedra de Ortega (Pcia. de Río Negro): producción, funcionalidad y tendencias temporales. Rastros. Arqueología e historia de la cuenca del río Limay. Serie monográfica, vol. 2.

Fernández, P. M., Bellelli, C., Carballido Calatayud, M., Podestá, M., \& Vasini, A. (2010). Primeros 
resultados de las investigaciones arqueológicas en el sitio Población Anticura (Río Negro, Argentina). En J. R. Bárcena, \& H. Chiavazza (Eds.), Arqueología argentina en el bicentenario de la revolución de Mayo Tomo V (pp. 1895-1900). Mendoza: Universidad Nacional de Cuyo.

Fernández, P. M., Carballido Calatayud, M., Bellelli, C., \& Podestá, M. (2013). Tiempo de cazadores. Cronología de las ocupaciones humanas en el valle del río Manso inferior (Río Negro). En A. F. Zangrando, R. Barberena, A. Gil, G. Neme, M. Giardina, L. Luna, C. Otaola, S. Paulides, L. Salgán, \& A. Tivoli (Eds.), Tendencias teórico-metodológicas y casos de estudio en la arqueología de Patagonia (pp. 167175). Buenos Aires: Museo de Historia Natural de San Rafael, Sociedad Argentina de Antropología e Instituto Nacional de Antropología y Pensamiento Latinoamericano.

Fernández, P. M., Carballido Calatayud, M., Bellelli, C., Podestá, M., \& Scheinsohn, V. (2011). Marcas en la piedra, huellas en la tierra. El poblamiento del bosque del suroeste de Río Negro-noroeste de Chubut. En S. Valverde, G. Maragliano, M. Impemba, \& F. Trentini (Eds.), Procesos históricos, transformaciones sociales y construcciones de fronteras. Aproximaciones a las relaciones interétnicas. Estudios sobre Norpatagonia, Argentina y Labrador, Canadá (pp. 195-221). Buenos Aires: Facultad de Filosofía y Letras, Universidad de Buenos Aires.

Fiore, D. (2011). Materialidad visual y arqueología de la imagen. Perspectivas conceptuales y propuestas metodológicas desde el sur de Sudamérica. Boletín del Museo Chileno de Arte Precolombino, 16(2), 101-119.

Fiore, D. (2012). Diseños y tempos en el arte mobiliar del canal de Beagle (Tierra del Fuego). Una exploración de los ritmos de cambio en la decoración de artefactos óseos. Relaciones de la Sociedad Argentina de Antropología, 37(1), 183-206.

Fiore, D., \& Borella, F. (2010). Geometrías delicadas. Diseños grabados en cáscaras de huevo de Rheidae recuperados en la costa norte del Golfo San Matías, Río Negro. Intersecciones en Antropología, 11, 277-293.

Forcelli, D. O. (2000). Moluscos Magallánicos. Guía de moluscos de la Patagonia y del sur de Chile. Buenos Aires: Editorial Pearson.

Franco, N. V., Guarido, A. L. Montenegro, T., \& Ambrústolo, P. (2012). Variabilidad en la utilización de pigmentos en entierros humanos del Holoceno tardío en la cuenca superior del río Santa Cruz (Patagonia argentina). Boletín del Museo Chileno de Arte Precolombino, 17(2), 11-25.

Gamble, C. (1982). Interaction and alliance in Palaeolithic society. Man, 17(1), 92-107.

Gómez Otero, J. (2006). Dieta, uso del espacio y evolución en poblaciones cazadoras -recolectoras de la costa centro- septentrional de Patagonia durante el Holoceno medio y tardio. (Tesis inédita de doctorado). Facultad de Filosofía y Letras, Universidad de Buenos Aires, Buenos Aires.

Goñi, R. (2000). Arqueología de momentos históricos fuera de los centros de conquista y colonización: un análisis de caso en el sur de la Patagonia. En Desde el País de los Gigantes. Perspectivas Arqueológicas en Patagonia (pp. 283-296). Río Gallegos: Universidad Nacional de la Patagonia Austral.

Gradin, C. (1999). Sobre las tendencias del arte rupestre en Patagonia argentina. En M. Tamagnini (Ed.), Segundas Jornadas de Investigadores en Arqueología y Etnohistoria del Centro-Oeste del país (pp. 85-99). Río Cuarto: Universidad de Río Cuarto.

Gradin, C. J., Aschero, C. A., \& Aguerre, A. M. (1976). Investigaciones arqueológicas en la Cueva de las Manos Estancia Alto río Pinturas (provincia de Santa Cruz). Relaciones de la Sociedad Argentina de Antropología, 10, 201-250.

Gurova, M., Bonsall, C., Bradley, B., \& Anastassova, E. (2013). Approaching prehistoric skills: experimental drilling in the context of bead manufacturing. Bulgarian e-Journal of Archaeology, 3, 201-221.

Gutiérrez Zugasti, F. I. (2008-2009). Análisis tafonómico en arqueomalacología: el ejemplo de los concheros de la región cantábrica. Krei, 10, 53-74.

Hajduk, A., Albornoz, A., \& Lescano, M. (2004). El Mylodon en el patio de atrás. Informe preliminar sobre los trabajos en el sitio El Trébol, ejido urbano de San Carlos de Bariloche, provincia de Río Negro. En M. T. Civalero, P. M. Fernández, \& A. G. Guraieb (Eds.), Contra viento y marea. Arqueología de Patagonia (pp. 715-732). Buenos Aires: Instituto Nacional de Antropología y Pensamiento LatinoamericanoSociedad Argentina de Antropología.

Hajduk, A., Albornoz, A. M. \& Lezcano, M. J. (2008). Arqueología del área del lago Nahuel Huapi. La problemática del uso del medio ambiente boscosolacustre cordillerano y su relación con el de estepa y ecotono vecinos. En P. Azar, E. Cúneo \& S. Rodríguez 
(Eds.), Tras la senda de los ancestros: Arqueología de Patagonia (pp.1-17).

Kuhn, S. L., \& Stiner, M. C. (2007). Body ornamentation as information technology: towards an understanding of the significance of early beads. En P. Mellars, K. Boyle, O. Bar-Yosef \& C. Stiner (Eds.), Rethinking the human revolution: new behavioural and biological perspectives on the origin and dispersal of modern humans (pp. 45-54). Cambridge: McDonald Institute for Archaeological Research.

Laporte, L., Desse-Berset, N., Gruet, Y., \& Tresset, A. (1994). Un lieu de fabrication de parure au Néolithique Final et économie de subsístanse. Le site de Ponthezières à Saint-Georges-d'Oléron (Charente-Maritime). En Le Néolithique du centre-ouest de la France. Actes du XXI colloque inter-régional sur le Néolithique (pp. 237-255). Poitiers.

Leonardt, S. (2014). Producción local de cuentas de valva en el bosque del Noroeste de Patagonia. Una aproximación desde la arqueología experimental. Relaciones de la Sociedad Argentina de Antropología, 39(2), 463482.

McDonald, J. (2008). Dreamtime superhighway: an analysis of Sydney Basin rock art and prehistoric information exchange. Terra Australis 27. Canberra: ANU E Press.

Onetto, M. (1986-1987). Nuevos resultados de las investigaciones en Campo Nassif 1 . Valle de Piedra Parada. Provincia del Chubut. Relaciones de la Sociedad Argentina de Antropología, 17(1), 95121.

Orchard, W. C. (1975). Beads and beadwork of the American Indians. New York: Museum of the American Indian, Heye Foundation.

Podestá, M. M., Albornoz, A. M., Vasini, A., \&Tropea, E. (2009). El sitio Peumayén 2 en el contexto del arte rupestre del bosque andino-patagónico. Comechingonia Virtual, 3(2), 117-153.

Prates, L. (2008). Los indígenas del Río Negro. Un enfoque arqueológico. Buenos Aires: Sociedad Argentina de Antropología.

Sanguinetti de Bórmida, A., \& Curzio, D. (1996). Excavaciones arqueológicas en el sitio Piedra de Águila 11. Praehistoria, 2, 43-100.

Scheinsohn, V. (2011). Rock art information among huntergatherers in Northwest Patagonia: an assessment of broadscale and territorial models. En R. Whallon, W. Lovis, \& R. Hithcock (Eds.), Information and its role in hunter-gatherers bands (pp. 235-247). Nuevo
México: Cotsen Institute of Archaeology Press.

Silveira, M. (1996). Alero Los Cipreses. En J. Gómez Otero (Ed.), Arqueología. Sólo Patagonia. Ponencias de las Segundas Jornadas de Arqueología de la Patagonia (pp. 107-118). Puerto Madryn: Centro Nacional Patagónico-Consejo Nacional de Investigaciones Científicas y Técnicas.

Silveira, M., López, L., \& Pastorino, G. (2010). Movilidad, redes de intercambio y circulación de bienes en el sudoeste del Neuquén (Norpatagonia, Argentina). Los moluscos marinos del lago Traful. Intersecciones en Antropología, 11, 227-236.

Stiner, M. C. (2014). Finding a Common Bandwidth: Causes of Convergence and Diversity in Paleolithic Beads. Biological Theory, 9(1), 51-64.

Suárez Diez, L. (2002). Tipología de los objetos prehispánicos de concha. México: Instituto Nacional de Antropología e Historia.

Taborin, Y. (2004). Langage sans parole. La parure aux temps préhistoriques. París: La Maison des Roches.

Velázquez Castro, A. (2007). La producción especializada de los objetos de concha del Templo Mayor de Tenochtitlan. México: Instituto Nacional de Antropología e Historia.

Velázquez Castro, A., \& Juárez Cossío, D. (2007). La colección de objetos de concha de Moral-Reforma. En A. Velásquez Castro, \& L. S. Lowe (Eds.), Los moluscos arqueológicos. Una visión del mundo Maya (pp. 6197). México: Universidad Autónoma de México.

Whallon, R. (2006). Social networks and information: Non"utilitarian" mobility among hunter-gatherers. Journal of Anthropological Archaeology, 25, 259-270.

White, R. (1993). A social and technological View of Aurignacian and Castelperronian Personal Ornaments in SW Europe. En V. Cabrera Valdes (Ed.), El origen del hombre moderno en el Suroeste de Europa (pp. 327-357). Madrid: Ministerio de Educacion y Ciencia.

Wiessner, P. (1983). Style and social information in Kalahari san projectile points. American Antiquity, 48(2), 253-276.

Wobst, M. (1977). Stylistic behavior and information exchange. En C. E. Cleland (Ed.), Pappers for the Director: Research Essays in Honor of James $B$. Griffin, Anthropological Papers 61 (pp. 317-342). Ann Arbor: University of Michigan.

Zubimendi, M. A. (2010). Malacological artifacts in Argentine Patagonia. Munibe. Suplemento, 262-270.

Zubimendi, M. A. (2015). Síntesis historiográfica de las investigaciones sobre artefacto arqueomalacológicos 
de la Patagonia Continental Argentina (18672011). En H. Hammond, \& M. A. Zubimendi (Eds.), Arqueomalacología. Abordajes metodológicos y casos de estudio en Cono Sur, (pp. 217-253). Buenos Aires: Fundación de Historia Natural Félix de Azara.

Zubimendi, M. A., \& Ambrústolo, P. (2011). La presencia de ítems marinos en el interior de Patagonia Central. En
A. Guiance (Ed.), Movilidad y migraciones. Actas de las III Jornadas Multidisciplinarias (pp. 291-305). Buenos Aires: Editorial Dunken.

Zubimendi, M. A., \& Moreno, J. E. (2014). La presencia de artefactos arqueomalacológicos en la localidad arqueológica Delta del arroyo Vulcana (lago Musters, provincia del Chubut). Intersecciones en Antropología, 15, 71-77. 
